\title{
Automated, real-time geohazard assessment in deep underground mines
}

\author{
WJ McGaughey Mira Geoscience Ltd., Canada \\ V Laflèche Mira Geoscience Ltd., Canada \\ C Howlett Mira Geoscience Ltd., Canada \\ JL Sydor Mira Geoscience Ltd., Canada \\ D Campos Mira Geoscience Ltd., Canada \\ J Purchase Mira Geoscience Ltd., Canada \\ S Huynh Mira Geoscience Ltd., Canada
}

\begin{abstract}
We introduce an automated, real-time geohazard assessment system designed specifically for underground mining. The system, which we call Geoscience INTEGRATOR, is based on the quantitative 4D geohazard computational strategy that we previously developed and reported on at the Seventh International Conference on Deep and High Stress Mining in Sudbury, Canada, in September 2014. The computational strategy relies on modelling multiple, independent hazard criteria related to geology, structure, rock mass condition, stress, seismicity, mine geometry, and support on the rock interface where the hazards occur, and building a statistical model that relates past hazard occurrence to the state of those criteria at the time of the hazard (McGaughey 2014). Further research and development has been carried out since then with the objective of developing an operational computational infrastructure that enables practical geohazard assessment using this approach. We have developed a system for automatically assembling and managing the required inputs, managing the geohazard assessment computational processes, and providing meaningful reports to mine operators. This has been accomplished within three recent Canadian research programs managed by the Centre for Excellence in Mining Innovation (CEMI): 'Smart Underground Monitoring and Integrated Technologies' (SUMIT), 'Mining Observatory Data Control Centre' (MODCC), and 'Ultra-Deep Mining Network' (UDMN). The initial system design objective under the SUMIT program was a capability to "provide researchers with user-friendly access to mine datasets and their contextual information to facilitate and optimise research efforts." The system was further developed in the ensuing projects to provide a powerful 3D visualisation interface and to support, track, and automatically report on $4 D$ dynamic mine models and associated geohazards in a deep operating mine context. It is now being deployed to manage multi-institution, multi-site data from both SUMIT and other major research projects in addition to implementations at operating mine sites to improve rockburst and other types of geotechnical hazard assessment.
\end{abstract}

Keywords: geohazard, rockburst, seismicity, machine learning, data management

\section{Introduction}

The three most important lessons of our previous work (McGaughey 2014) in the integration of multiple data types for quantitative geohazard assessment, carried out in many different mine settings across a range of geohazards (e.g. fault-slip rockbursts, strainbursts, roof fall, slope failure, water inflow) are:

- Geohazard assessments that integrate many data types are demonstrably more effective than assessments that rely heavily on a single data type; in particular, they are more effective than relying on microseismic monitoring data alone. 
- Numerical weightings through which disparate data are combined into an overall geohazard assessment are better determined by data-driven, statistical back-analysis than by expert, knowledge-driven, manual determination.

- Operations do not typically have the data management infrastructure to provide the required inputs for integrated analysis of multiple data types. This situation is manageable where hazard assessment updates are only required offline on a one-off or intermittent basis. On the other hand, a more useful continuous or real-time operational assessment of hazard requires up-to-date access to all the data types required for calculation, including microseismic and other monitoring data. Lack of continuous, universal data access precludes optimal, timely geohazard assessment.

This paper describes our work in creating a data management infrastructure that manages multiple streams of time-dependent 3D input data, including seismicity, stress, ground deformation, other types of geotechnical monitoring, rock mass properties, geological models, ground support, mine geometry, mine operations, and incident reports. The result is that multi-disciplinary data are queried, visualised and, most importantly, automatically integrated into a single data structure. A single data structure supporting all the hazard-relevant data is required by machine learning or conventional statistical analysis for determination of the associations and statistical patterns that reveal relationships amongst data and hazard incidents. Reports may then be manually generated or automatically dispatched.

\subsection{Geotechnical data management in mines}

An integrated, coherent system for managing multi-disciplinary data is required if teams are to share data, models, and interpretations for optimal geotechnical decision making. In our experience, Microsoft Office ${ }^{\circledR}$ products are used ubiquitously in the mining industry to manage and communicate day-to-day geotechnical information - Excel ${ }^{\circledR}$ for manipulation of numbers and PowerPoint ${ }^{\circledast}$ for communication of ideas and results. Neither of these products were created to manage or interpret data and are consequently unsuitable for those purposes in many ways. This is because data formats and file storage systems using such tools are idiosyncratic, typically understood by the individual data custodian rather than colleagues or the organisation, even when the intention is that data be accessible to all stakeholders. Standardisation of data structures is necessary for confidence in data that is shared; and data that is accessible and clearly understood is prerequisite to integrating multiple data streams into geohazard assessment framework. Although drillhole, production, and microseismic databases are commonplace, they generally exist in independent repositories, separate from each other and from other key data repositories such as geotechnical instrumentation or mine drawing and survey files. Although there are exceptions, such as the cave management system described by Mol et al. (2012), comprehensive systems and associated best practices to manage an organisation's geotechnical project data, interpretation, and decisions are generally not employed. The result is that geotechnical interpretations that require, or should be validated by, multiple data types are difficult to carry out in practice. Not only must primary data be located and exported from multiple systems, but contextual metadata can be uncharacteristically ad hoc and incomplete.

The need for a system to support, record, and communicate multi-disciplinary geotechnical interpretation is pressing as mines become deeper, geotechnical problems become more acute, and the flow of data available for analysis has ever-greater speed and volume. Geotechnical hazard assessment, such as rockburst risk evaluation and monitoring, cannot be effectively addressed in a timely manner without access to a multi-disciplinary 4D data management system. This is because geotechnical hazard evolves over time, the underlying data are time-dependent, and the results of analysis must be routinely updated. The Sudbury-based Centre for Excelling in Mining Innovation's (CEMI) 'Smart Underground Mining and Integrated Technologies' (SUMIT) research program (Katary 2016), which involved participation from multiple mine sites, universities, and researchers, anticipated the need for a data management system that could support analysis of complex problems that required collaborative interpretation of multi-disciplinary 4D underground mine data and associated contextual metadata. Geoscience INTEGRATOR, the system described here, was initially developed to answer the SUMIT program mandate. 


\section{The geohazard assessment problem}

We have carried out many geotechnical hazard assessments for different classes of geohazard at a wide range of mine types. This allowed us to successfully develop a model-based approach in which individual criteria related to the hazard in question are captured in a spatial mine model and then used as variables to be combined in a quantitative analysis. Modelling the hazard criteria can be challenging, not the least because many of the key criteria, and thus the resulting models, are spatially 3D and time-dependent. Taking advantage of recent advances in machine learning or statistical data integration requires that the individual hazard criteria be co-located in space and time, on the modelled rock interfaces where hazards may occur, so that relationships amongst them may be meaningfully investigated. In practice, we handle this by modelling each of the hazard criteria on the mine development (drift, stope, or slope in the case of open pits), and account for the 4D nature of the problem by time-stepping 3D models. The modelling requires application of methods that map how data such as structure, rock quality, and seismicity, which is often located far into the rock mass away from development, manifest hazard within the mine development. The approach, including the advantages of a quantitative, statistical back-analysis approach versus manual setting of criteria weights, is described in detail through a case study by McGaughey (2014).

An important lesson from these studies is that operations typically do not have the data management infrastructure that readily provides the required data to a comprehensive, multi-disciplinary $4 \mathrm{D}$ analysis. This situation is manageable where hazard assessment updates are only required offline on an intermittent basis. Data compilation is always the most expensive and time-consuming part of this type of project, particularly when there is a lot of multi-disciplinary data and several custodians involved. It is our experience that if projects falter, they typically falter at this stage, which is a symptom of inadequate data management practices. Ongoing or real-time operational assessment of hazard requires up-to-date access to all the data types required for calculation, including microseismic and other monitoring data.

\section{System requirements and design summary}

The SUMIT research program foresaw similar data management challenges, with its multiple case study sites, widely differing experiment types, and multiple research institutions and collaborators. A need was cited by the original scoping documents for "creating an appropriate structure for collecting, standardising, storing, and sharing mining datasets with research and development entities in a fully controlled manner." This research need precisely mirrors the wider industry need for such a system as the foundation of strategies for interpretation and operational decision making when confronted with complex phenomena such as rockbursts in underground mines.

The original scoping document outlines the requirements of the SUMIT data management system as follows (Katary 2016):

An important part of this [system] is to provide SUMIT researchers with user-friendly access to mine datasets and their contextual information in order to facilitate and optimise research efforts. The data and contextual information can vary from researcher to researcher. Equally important, the solution platform must offer a single point of access that minimises demand on mining personnel by eliminating duplicate data requests.

The datasets that are needed for this project are [currently] created and stored by mining companies in disparate data management systems. An important component of the project is to put in place the protocols, adapters, and converters that bring these datasets in the data warehouse in consistent formats and to update them on a regular basis. 
The following tools are envisaged:

- Topical data browsing

- Data querying/filtering

- Visual data browsing and querying

- Downloading

- Posting back research results.

For the SUMIT project, the following mine datasets are envisaged:

- Mine infrastructure, i.e. mine openings

- Seismic events catalogue

- Seismic triggered waveforms

- Geology data (mapped and interpreted)

- Drill hole logging data

- Plant activity

- Mobile equipment activity

- Extensometer measurements

- Blast reports.

A graphical summary of the required system from the original scoping documents is presented in Figure 1.

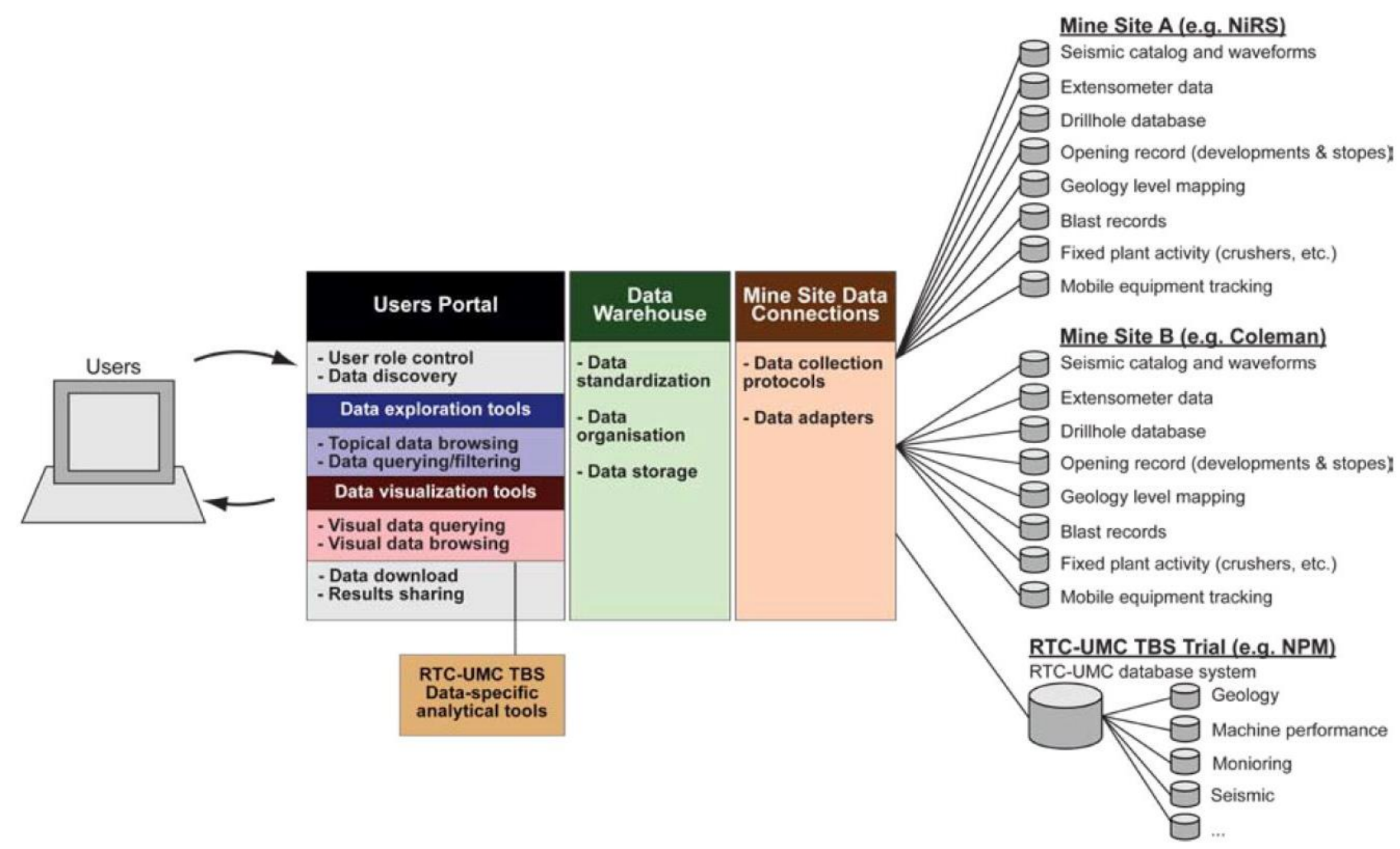

Figure 1 Proposed data management system architecture and components, from the original SUMIT project scoping documents 
We defined the following system design requirements to meet the initial objectives:

- Data is organised by mine site (or 'project') and data type.

- A permissions system defines which users have access rights to read, write, view, and download data.

- All data have the capability of time-variance, stored either as [data value, time] or [data value, start time, end time].

- Although allowable data types are system-defined, data formats are user-defined.

- A file and document management system enables linkages between data and associated files (such as image or other binary files) and documents.

- A 'tagging' system is used to label and query data.

- 3D volumes (e.g. drifts, stopes, geological domains) can be defined and data that are within those volumes will be automatically recognised to facilitate query.

- Time intervals can be defined independently of date to facilitate time-of-day query such as shifts.

- The primary system interface is a web browser that can be used on a desktop or tablet device.

- A powerful, desktop 3D visualisation application is used for querying and viewing data in the 3D mine context.

- A reporting system allows reports to be generated on user demand or according to a schedule, in which case the reports are automatically created and emailed to a specified user group.

- Real-time data updates are accommodated either by monitoring specified network file system folders for new data files or directly accessing data from other relational database systems. When availability of new data is detected from either of these sources, the new data are automatically imported after application of quality control procedures define for each data type. When new data are attempted to be imported, a notification is sent to system administrators describing the import data and whether the import action succeeded or failed.

- The system contains an application programming interface (API) that allows third-party applications to communicate with it.

A system that meets the design requirements has been successfully developed and deployed. Figure 2 presents a general system schematic, showing key components including the back-end database server, the mail server for report distribution, the web browser client as desktop and tablet application, the 3D visualisation and query interface ('Geoscience ANALYST'), and the main Geoscience INTEGRATOR server at the centre of the system.

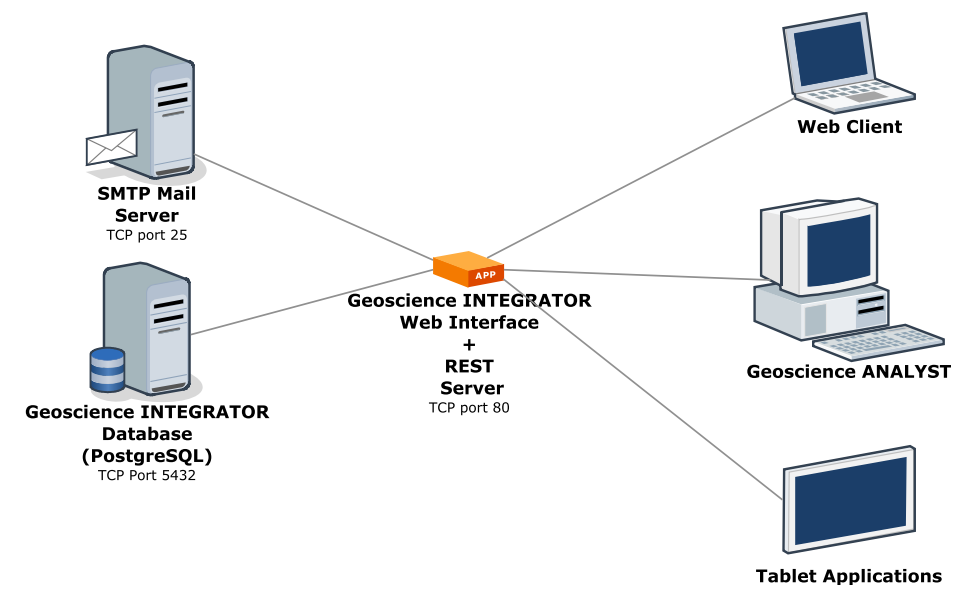

Figure 2 Geoscience INTEGRATOR system schematic 
Figure 3 is a screen image from the Geoscience ANALYST interface showing relative rockburst hazard on underground mine workings. Figure 4 illustrates the intended deployment configuration, with one or more mine sites continuously supplying data to a single server based onsite, regionally, or cloud-hosted. In each of these cases the server will typically sit behind the operating entity's firewall for security.

Development of the system foundation under the SUMIT project led to two other CEMI research initiatives that have advanced the technology. These were 'Mining Observatory Data Control Centre' (MODCC), and '4D Real-Time Geotechnical Hazard Assessment and Reporting for Ultra-Deep Mining', a project within the Ultra-Deep Mining Network (UDMN), completed in December 2016. These additional projects enhanced the original SUMIT system with additional data themes, advanced 3D visualisation, real-time geohazard computation and an experimental 'learning' capability; a capability that automatically adapts the hazard assessment computations in response to changes in the mine, as discussed in the following section.

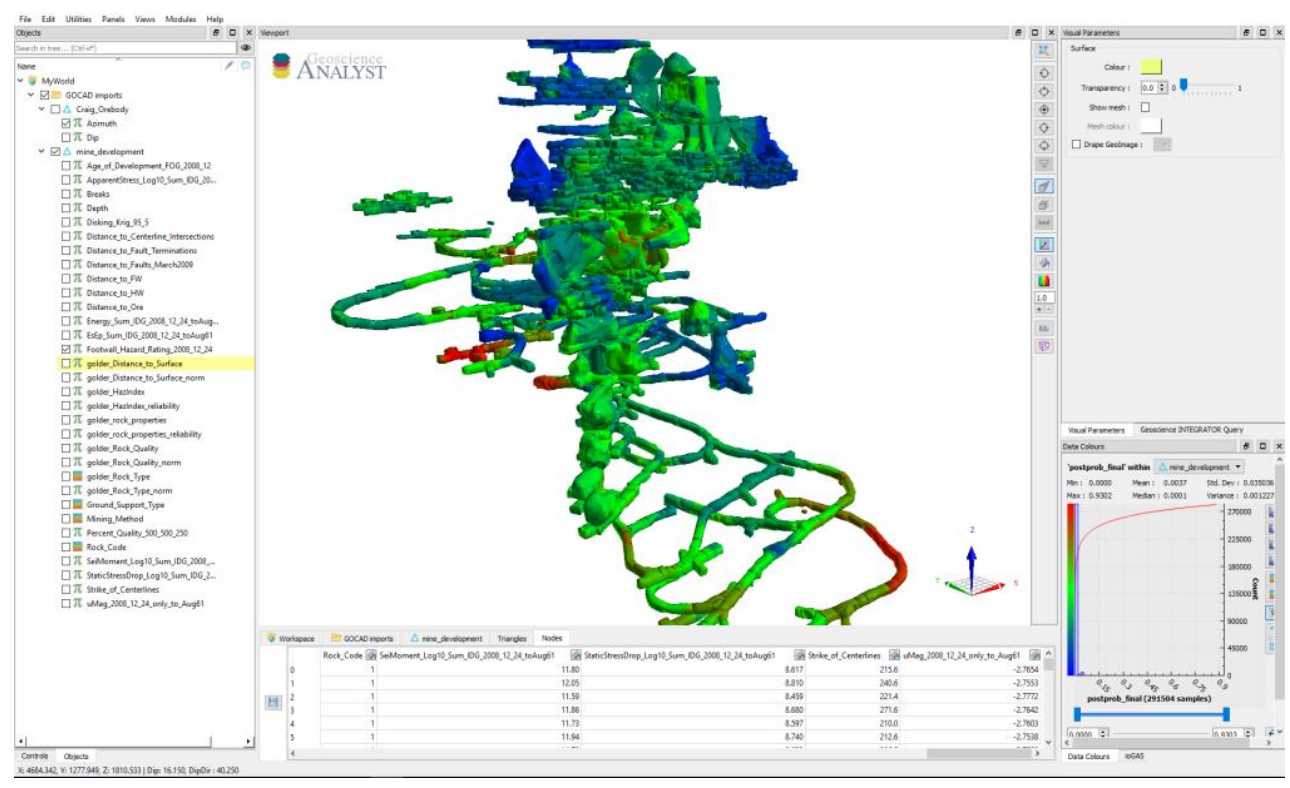

Figure 3 The Geoscience ANALYST desktop interface used for 3D visualisation and query of the data management system, depicting mine infrastructure coloured by relative hazard, in this case with red meaning high rockburst hazard, green moderate, and blue low

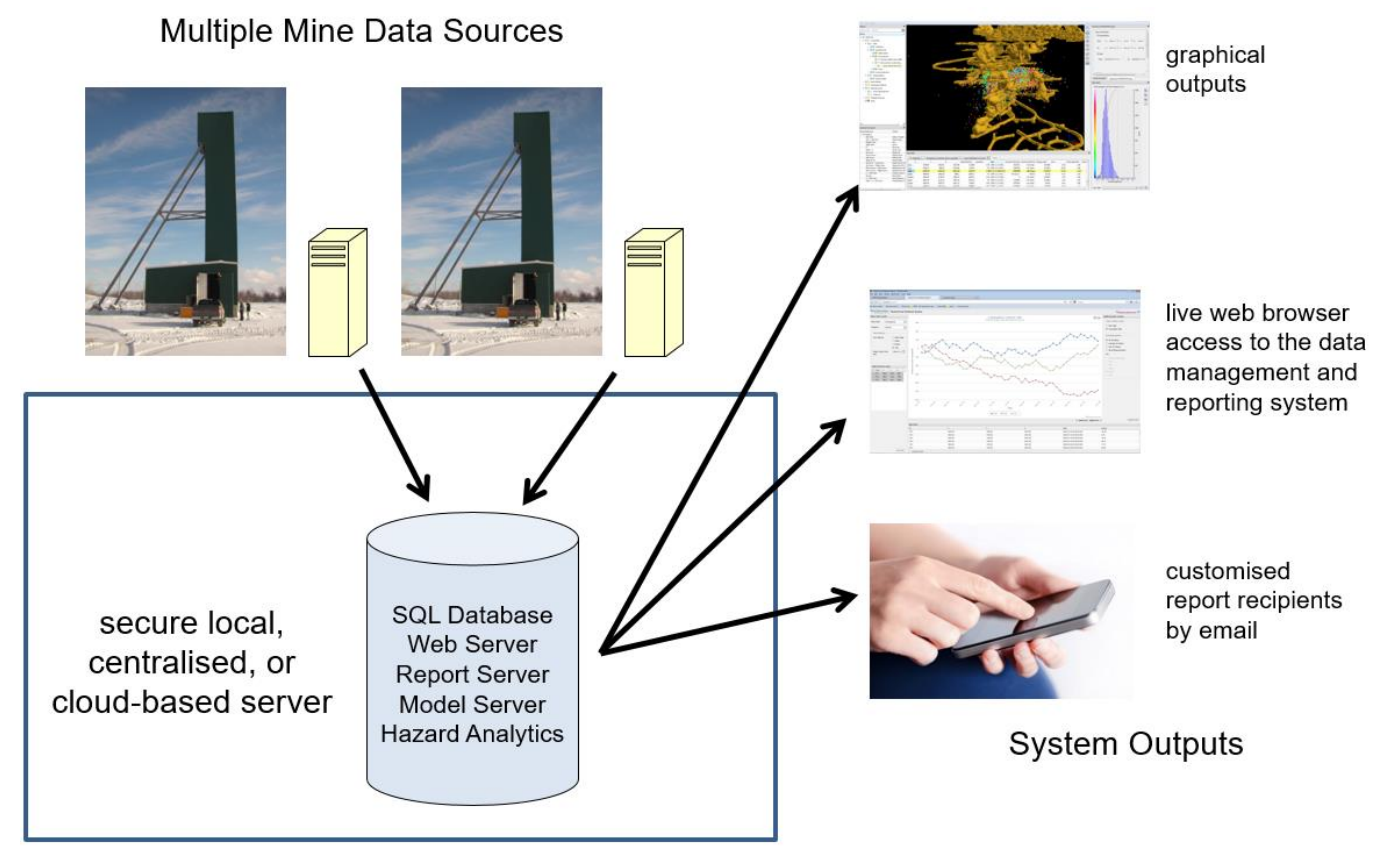

Figure 4 System deployment configuration schematic 


\section{$4 \quad$ Towards a 'living, learning' approach to geohazard assessment}

With a system in place to manage time-dependent 3D mine data and contextual metadata with continuous data feeds, the stage is set for real-time geohazard assessment. We are taking a data-adaptive approach to geohazard assessment in the UDMN R\&D project, with the following two key features:

- The mine and rock mass are represented within the system as 'living' models, consistent with current data and interpretation, keeping a record in time of the state of the mine and its rock mass environment. In practice this means that the appearance of new drillhole or other data in the system will automatically trigger (if desired) a re-computation of the rock mass or other model parameters. For example, new rock quality data in the drillhole database may trigger re-kriging of rock quality parameters in the rock mass block model, which in turn provides an updated standard input hazard criterion. The same concept holds for mine infrastructure, with drifts, stopes, and other mine geometry information updated as it becomes available.

- As mining progresses in time, the statistical relationships amongst hazard criteria and hazard occurrence may themselves change in response to new mechanisms coming into play as depth, stress regime, or other key drivers change. Because the data management system maintains a record of hazard criteria and hazard occurrence as a function of space and time, the statistics of the relationships amongst these parameters can be evaluated over time, in effect resetting the weighting factors that determine how the integrated hazard assessment is computed. Thus, the system continuously 'learns' about dynamically changing mine conditions and how they relate to hazard.

To accomplish the 'living, learning' system, we have added two additional components to the original Geoscience INTEGRATOR system shown in Figure 2 - a modelling server and an analytics server. These are illustrated schematically in Figure 5. The model server is the GOCAD ${ }^{\circledR}$ Mining Suite (Paradigm 2017), a commercially-available modelling engine, present on the server as a run-time engine only, without the standard user interface, that can carry out advanced geological modelling and spatial query computations in response to commands from Geoscience INTEGRATOR. This sub-system provides the 'living' model capability in response to availability of new data. An example is computation of volumetric seismic event density over a specific time window as new data become available. The analytics server is a dedicated subsystem that operates on the standardised data structure that the data management system maintains of hazard criteria and hazard occurrences over space and time.

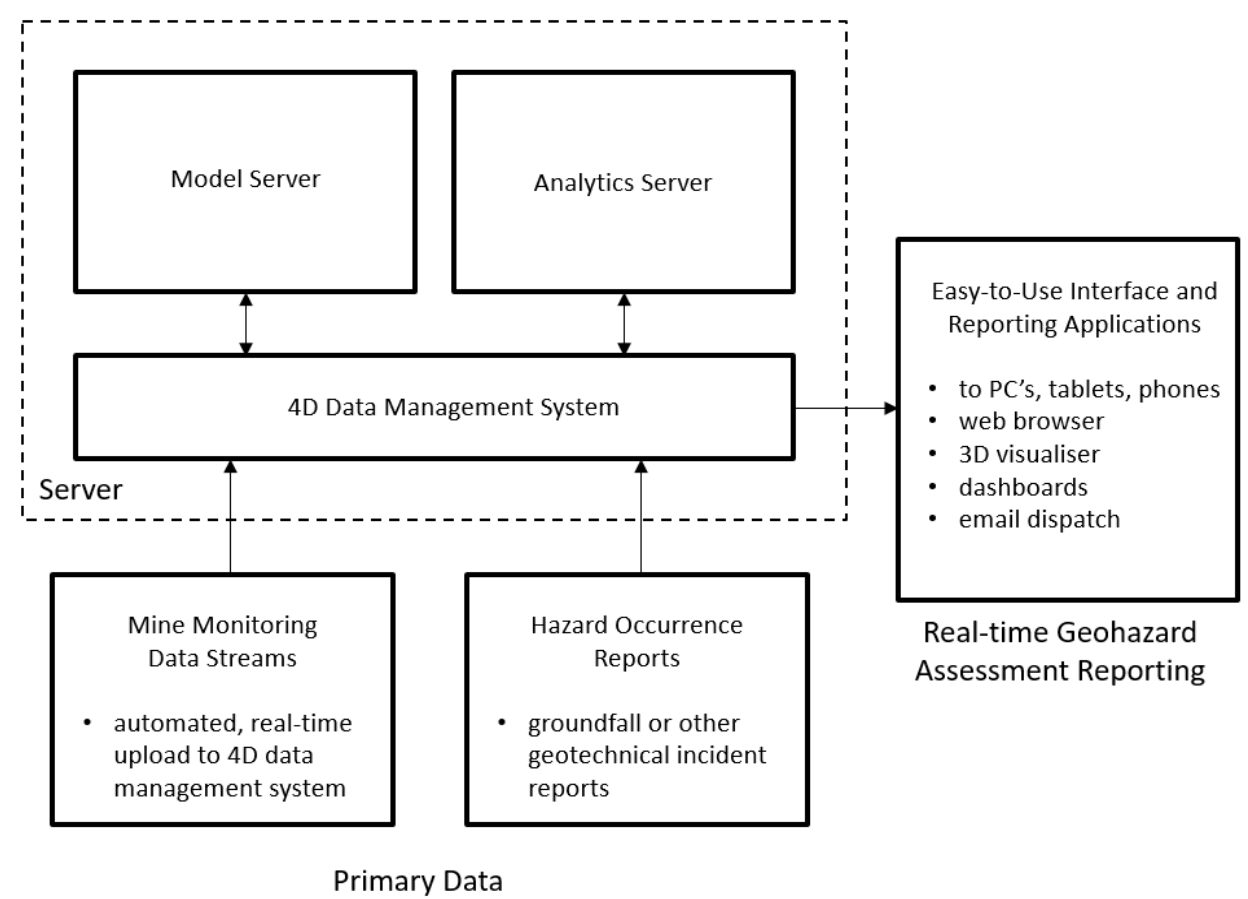

Figure 5 Advanced Geoscience INTEGRATOR system schematic as developed under the UDMN program 
Our previous studies in rockburst-prone deep mining environments clearly demonstrated that optimum geohazard assessment depends on access to a representative rock mass model that includes the 3D distribution of rock type, major structures, joint distribution and characteristics, rock quality, discing, and stress. Rock mass model parameters of interest may also include intact rock strength, strength and stiffness at the rock mass scale, and other variables such as seismically-derived energy release and damage parameters. Some of these variables, such as stress and seismically-derived parameters, change continuously in time as mining proceeds. Others such as rock type or the 3D distribution of rock quality and disking are either time-invariant or change slowly. Even if some characteristics of the rock mass (such as rock type or major structure geometry) do not change as mining progresses in the actual earth, our interpretation of these parameters may change as more data becomes progressively available. In that sense, all aspects of the rock mass model should be considered to evolve in time.

At the core of the living rock mass model is a multi-scale structural and geological model that integrates data from the regional scale to the detailed underground observation scale. Understanding structure at the regional scale, based on geological and geophysical data, is important for assessing the possibility of catastrophic seismic release on major structures. At the local scale, it has been demonstrated in previous geohazard studies that proximity to structures and dykes, proximity to structural intersections, local joint orientation, major structure geometry, and fault slip tendency (a function of local structural geometry and stress) all have an important influence on geohazard. The 3D distribution of rock type, rock quality and discing are similarly important. Microseismic event density and other parameters derived from microseismic data, such as stress information, provide the other important geohazard-related characterisation of the evolving rock mass. Microseismic event density computation, 4D clustering analysis, and $3 \mathrm{D}$ interpolation of source parameters that have proven to be correlated to geohazard in previous studies $\left(E_{s} / E_{p}\right.$ ratio for example) are automatically and continuously updated as rock mass model attributes using the capability of the model server integrated with the database.

\section{$5 \quad$ Concluding remarks}

Geoscience INTEGRATOR, the data management and collaboration system initially developed through the SUMIT program, is being successfully deployed for R\&D and industry mine site applications. Its unique capability to bring together vast amounts of multi-disciplinary 4D data is creating opportunities to analyse and interpret data in new ways, including the promise of a 'living, learning' system. This directly adds value for mine operators by providing decision-making tools for a more accurate and timely assessment of geohazard.

\section{Acknowledgement}

We gratefully acknowledge the ongoing support of the Centre of Excellence in Mining Innovation (CEMI), particularly through Damien Duff (Vice President - Geoscience \& Geotechnical R\&D) and Glencore Canada, particularly through the ongoing support of Brad Simser (Senior Ground Control Engineer).

\section{References}

Katary, S 2016, 'SUMIT program presents results: mining deeper, safer and more economically', Centre for Excellence in Mining Innovation, viewed 2 September 2016, https://www.miningexcellence.ca/?p=4269/

McGaughey, WJ 2014, '4D data management and modelling in the assessment of deep underground mining hazard', in M Hudyma \& Y Potvin (eds), Proceedings of the Seventh International Conference on Deep and High Stress Mining, Australian Centre for Geomechanics, Perth, pp. 93-106.

Mol, M, van As, A, Tosen, R \& McGaughey, WJ 2012, 'The design and development of an integrated cave management system', Proceedings of MassMin 2012: 6th International Conference \& Exhibition on Mass Mining, Canadian Institute of Mining, Metallurgy and Petroleum, Sudbury.

Paradigm 2017, GOCAD ${ }^{\circledR}$ Mining Suite, software, Paradigm, viewed 24 January 2017, www.pdgm.com/solutions/ data-management-and-interoperability/development-kits/ 